\title{
DESIGN OF PMOLED DISPLAY SYSTEM BASED ON PAM
}

\author{
Jianying Shi ${ }^{*}$, Yanbin $\mathrm{Xu}^{2}$ \\ Corresponding author:Jianying Shi \\ ${ }^{1}$ Electronic Information Engineering College, HeBei University, BaoDing, HeBei 071002, China; \\ ${ }^{2}$ Department of Electrical Engineering, Hebei College of Science and Technology, BaoDing HeBei 071000, China \\ E-mail: jianyingshieie@163.com
}

\begin{abstract}
As the next generation of flat panel displays, OLED displays have a variety of outstanding features and advantages. At present, OLED display technology is a hotspot in the display technology research in the world. In addition, there are a large number of small and medium size OLED displays used in portable devices, and the manufacturing technology of large-size OLED display is maturing. An image display control system is designed for the PMOLED display. The system uses dedicated PMOLED display driver chip packaged by TAB and have advantages of easy driving and high reliability. Oxide TFT has advantages of better mobility, stability and uniformity, which is one of the most powerful competitors of active substrate technology. In this paper, a video display control system based on AMOLED display driver circuit of metal oxide TFT substrate is designed. The system uses TFT-LCD driver chip of COG package in place of AMOLED display dedicated driver chip, which has advantages of easy achievement and cheap and meets the needs of initial research and development of AMOLED display. The system takes DVI interface as the video signal transmission interface, and takes FPGA as a scan and data driver chip timing controller with reusable and flexible features. Video display control system has achieved a 7 -inch $320 \times 3 \times 240$ pixel AMOLED display real-time dynamic video display with 64 grayscale and $60 \mathrm{~Hz}$ frame rate, and the display is good.
\end{abstract}

Keywords: PMOLE, AMOLED, MCU, FPGA, DVI.

\section{Introduction}

Organic Light Emitting Diode is also known as Organic Light Emitting Display, referred to as OLED. Under the effect of electric field, the hole and electron will enter the organic material layer from the cathode and anode. After the production of composite exciton, the phenomenon of radiation transition light can generate the OLED display device. As the latest third-generation display technology, it is considered as the most promising new display technology in the next few decades. The current OLED display technology still stays in the small-size screen development stage. Therefore, the research and application of small size OLED display becomes the necessary work in the existing situation. Compared to other types of OLED display, PMOLED display is more suitable for small size display. In view of this, this paper researchs the PAMLED display driver system based on the PAM (pulse amplitude adjustment).

\section{Literature Review}

According to the relationship between the drive circuit and the substrate, the organic light display can be divided into passive matrix OLED (PMOLED) and active matrix OLED (AMOLED), which is also known as passive drive OLED and active drive OLED. Generally, PMOLED organic light display drive uses common-cathode structure, which means the electrode connected to the cathode and the column electrode connected to the signal electrode. In the entire display system, positive voltage is applied to the corresponding column of luminescence pixels, and the corresponding column that does not require luminescence pixels is grounded. For the circuit, the row driving circuit is used for the cyclic scanning of the row electrodes so that the row electrodes are sequentially low. However, the column driving circuit latches the display data of the row and feeds back to the row electrodes under the control of the synchronizing signal. For how to reduce the power consumption and efficiency of PMOLED display driver chip, this paper explores domestic and foreign research from two aspects [1].

\section{(1) Pixel driving mode}

The so-called display brightness perceived by human eyes refers to the average brightness below screen display cycle, and the adjusting of screen brightness is to adjust the average. In addition, we can reach the 
purpose of adjusting e average brightness by changing the pulse width of drive current. The change of pulse width involves two modulation methods: one is the pulse amplitude modulation (PAM), which means the main value adjusted by the control of the time duration of current [3]. The other one is the width modulation (PWM), which means the main value adjusted by controlling the size of the current amplitude.

\section{(2) Manufacturing technique of analogue integrated circuit}

At present, the design of analog integrated circuit is mainly based on three techniques: standard bipolar technique, silicon gate CMOS technique and simulation BiCMOS technique. The traditional bipolar technique and single CMOS technique have the advantages of high speed, high current driving ability and high simulation precision. However, there is an urgent problem to be solved in power consumption and integration [2]. BiCMOS technique not only has the advantages of high integration and low power consumption of CMOS circuit, but also gets the advantages of bipolar circuit high-speed, high current drive capability.

\section{PMOLED Display Driver System Based on PAM}

\subsection{Design of BiCMOS bandgap reference source}

The reference source provides accurate drive current and voltage for the pixel, which directly determines the stability of the entire PMOLED display system.

\section{1) The achievement of the reference voltage with zero temperature coefficient.}

The main idea of the design of the bandgap reference is to use the inverse ratio characteristics between emitter voltage (bipolar transistor's base) and the absolute temperature, and use the inverse ratio characteristics between Emitter voltage differences (the base of two bipolar transistors) and the absolute temperature. After the linear superposition of these two voltages, we can get the reference voltage with zero temperature coefficients [4].
For bipolar transistors, the relationship between the base current IC and the base-emitter voltage $U_{B E}$ is:

$$
I_{C}=I_{S} \exp \left(\frac{U_{B E}}{U_{T}}\right)
$$

In the formula, $U_{T}=k T / q, \mathrm{k}$ is Boltzmann constant, q is electron charge and $I_{S}$ is transistor saturation current.

Since the saturation current $I_{S}$ is proportional to $\mu k T n_{t}^{2}, \mu$ represents minority carrier mobility, $n_{t}$ is the intrinsic carrier concentration of silicon. Formula (1) takes the derivative of T, and then the baseemitter voltage of the bipolar transistor is inversely proportional to the temperature T. If two of the same collector current of bipolar transistor bias are $I_{s 1}$ and $I_{s 2}$. Ignoring the base current, the direct proportion relationship between base-emitter voltage difference and temperature is:

$$
\frac{\partial \Delta U_{B E}}{\partial T}=\frac{\partial\left(U_{T} \ln \frac{n I_{0}}{I_{s 1}}\right)}{\partial T}=\frac{k}{q} \ln n>0
$$

According to the formula (2), when $U_{B E}=0.75 \mathrm{~V}, T=300 \mathrm{~K}$ the base emitter voltage of bipolar transistor meets the relationship between $\partial U_{B E} / \partial T=1.8 m V /{ }^{\circ} \mathrm{C}$

and

$$
\partial U_{T} / \partial T \approx 0.086 m \mathrm{~m} / \mathrm{C}
$$

$C$. Then we get the reference voltage of zero temperature coefficient.

$U_{r e f}=U_{B E}+17.2 U_{T} \ln n$

\section{2) Typical structure analysis of BiCMOS bandgap reference}

The characteristics of bandgap reference circuit have a simple structure and low noise. Like all bandgap reference sources, this kind of circuit also need a PTAT current source generated by PTAT voltage through resistance. The bias current of circuit is shared with the bandgap resistor R2 [5]. After adding the PTAT voltage into the base-emmiter of $Q_{2}$, we can get a stable bandgap reference voltage, and the loop gain is:

$$
A_{L}=\frac{-\mathrm{g}_{m} Q_{1}}{1+\mathrm{g}_{m} Q_{1} R_{1}} \cdot \frac{1}{\mathrm{~g}_{m} M_{1}}\left(-\mathrm{g}_{m} Q_{1}\right) \cdot \frac{1}{\mathrm{~g}_{m} M_{2}}=\frac{1}{1+\mathrm{g}_{m} Q_{1} R_{1}}
$$

If $Q_{1}$ is a bipolar transistor connected to a diode, the loop gain of the circuit becomes:

$$
A_{L}=-\mathrm{g}_{m} Q_{2} \bullet \frac{1}{\mathrm{~g}_{m} M_{2}} \bullet\left(\mathrm{g}_{m} M_{1}\right) \bullet\left(\frac{1}{\mathrm{~g}_{m} Q_{1}}+R_{1}\right)=1+\mathrm{g}_{m} Q_{2} R_{1}
$$


In the formula, $g_{m}$ is the transconductance of MOSFET.

It can be seen from the formula (5) that the circuit will be in the open-loop unstable state, so the bipolar transistor with diode connection cannot be on the same side as the resistance. Otherwise, it will cause the circuit to be unstable. The lack of the circuit shows that the bandgap reference source is greatly influenced by the external supply voltage, while the lack of an effective start circuit cannot make the circuit in normal working condition.

The emitter follower and the operational amplifier avoid the second order effect of the circuit output impedance and the base current, and do not cause the circuit to produce higher low frequency noise and larger of the voltage fluctuation. However, the extra use of operational amplifiers will doubly consume the bias current, and the offset voltage reaching up to several tens will greatly limit the accuracy of output reference current. Meanwhile, the higher output impedance $Z_{\text {out }}=r_{0}$ at the voltage reference output also reduces the driving ability of circuit. To further improve the temperature performance and accuracy of the circuit, Brokaw bandgap reference source contains longitudinal bipolar transistor (PNP, NPN) in the traditional CMOS process [6].

It can be seen from the figure that there is still an Erlie effect (base width modulation effect) in the circuit, which leads to the fact that the collector current of the bipolar transistor $Q_{1}$ and $Q_{2}$ in the current mirror can not be completely equal and the accuracy of the bandgap reference source is reduced.

Due to the influence of the Erlie effect $Q_{4}$ in the current mirror, the effective region of base is narrow and the depletion region is wide. Then the depletion region width $W_{D}$ of $Q_{4}$ and the effective base width $W_{\text {eff }}$ are:

$$
\begin{gathered}
W_{D}=\sqrt{\frac{2 \varepsilon}{q}\left(U_{T} \ln \frac{N_{B} N_{C}}{n_{i}^{2}}+U_{B C}\right)\left(\frac{1}{N_{B}}+\frac{1}{N_{c}}\right)} \\
W_{e f f}=W_{D} \bullet \frac{N_{C}}{N_{B}+N_{C}}
\end{gathered}
$$

In the formula, $\varepsilon$ is dielectric constant, $N_{B}$ and $N_{C}$ are the base and the doping concentration of collector. $U_{B C}$ is base-collector voltage difference, and $\mathrm{n}_{i}^{2}$ is the intrinsic carrier concentration. At that time, the collector current of $Q_{4}$ should be corrected to:

$$
I_{C}=I_{S} \bullet e^{\frac{U_{B E}}{U_{T}}}\left(1+\frac{U_{C E}}{U_{A}}\right)
$$

In the formula, $U_{A}$ is Early voltage with a range from $50 \mathrm{~V}$ to $150 \mathrm{~V}$.

\subsection{The design of non-curvature- compensated bandgap reference and current source}

\section{1) The design of core circuit}

In view of the defects on high output impedance of the traditional CMOS reference source and the Erlie effect in the Brokaw bandgap reference source, this design proposes a new design scheme, which can quickly adjust the fluctuation of the output reference voltage and eliminate the second order effect from output reference and base current. It has a low-frequency noise and high accuracy of the reference voltage [7].

In this paper, the PAM-based PMOLED display drive system, the bandgap reference current source circuit is shown in Fig. 3.

$Q_{1} 、 Q_{2} 、 R_{1} 、 R_{2}$ constitute the core circuit. In addition, $Q_{1}$ and $Q_{2}$ are used to ensure that their corresponding collector voltages are equal. In the design of reference source, the same potential in $\mathrm{X}$ and $\mathrm{Y}$ point is the foundation for improving the accuracy of reference voltage, that is to reduce the circuit's Erlie effect. In order to achieve this goal, the cascode current mirror is usually used to make the $\mathrm{X}$ and Y points have the same potential. However, this method increases the chip area and complexity of circuit design. This design uses a new reference voltage output stage, which uses $Q_{5}$ and $M_{1}$ instead of the emitter follower in traditional bandgap reference circuit as the output stage of the bandgap reference, where the emitter follower $Q_{5}$ ensures that the $\mathrm{X}$ and $\mathrm{Y}$ potentials are exactly equal. This method is conducive to match the PTAT current. And then a more accurate reference voltage can be obtained. Meanwhile, the source follower $M_{1}$ is used as a voltage reference output level to greatly reduce the output impedance of the circuit, so that the drive current can change following the output load (branch number of reference voltage copy). After this, we can achieve the purpose of stabilizing circuit output [8]. The transistor $M_{1}$ is a common drain amplifier and the output impedance of core circuit is:

$$
Z_{\text {out }}=\frac{R_{4}+R_{5}}{g_{m}\left(R_{4}+R_{5}\right)+1} \approx \frac{1}{g_{m}}
$$


According to Fig. 1, we can see that the bandgap reference voltage $U_{r e f}$ is obtained through resistance $R_{4}$ and $R_{5}$ partial pressure.

The base current of bipolar transistor $Q_{1}$ and $Q_{2}$ will flow $R_{4}$, resulting in the rise of base voltage and bandgap reference voltage. Based on this, the premise of eliminating the error is to keep the stablity of base voltage.
In this paper, the mehtod of compensating resistance is used to correct the base current of $Q_{2}$ without considering the base current of $Q_{1}$ and $Q_{2}$. The reference voltage is:

$$
U_{r e f}=\left(\frac{U_{T} \ln n}{R_{1}} R_{2}+U_{B E 2}\right) \frac{1}{R_{5}}\left(R_{4}+R_{5}\right)
$$

In the formula, $\mathrm{n}$ is the area radio of the emitter $Q_{1}$ and $Q_{2}$.

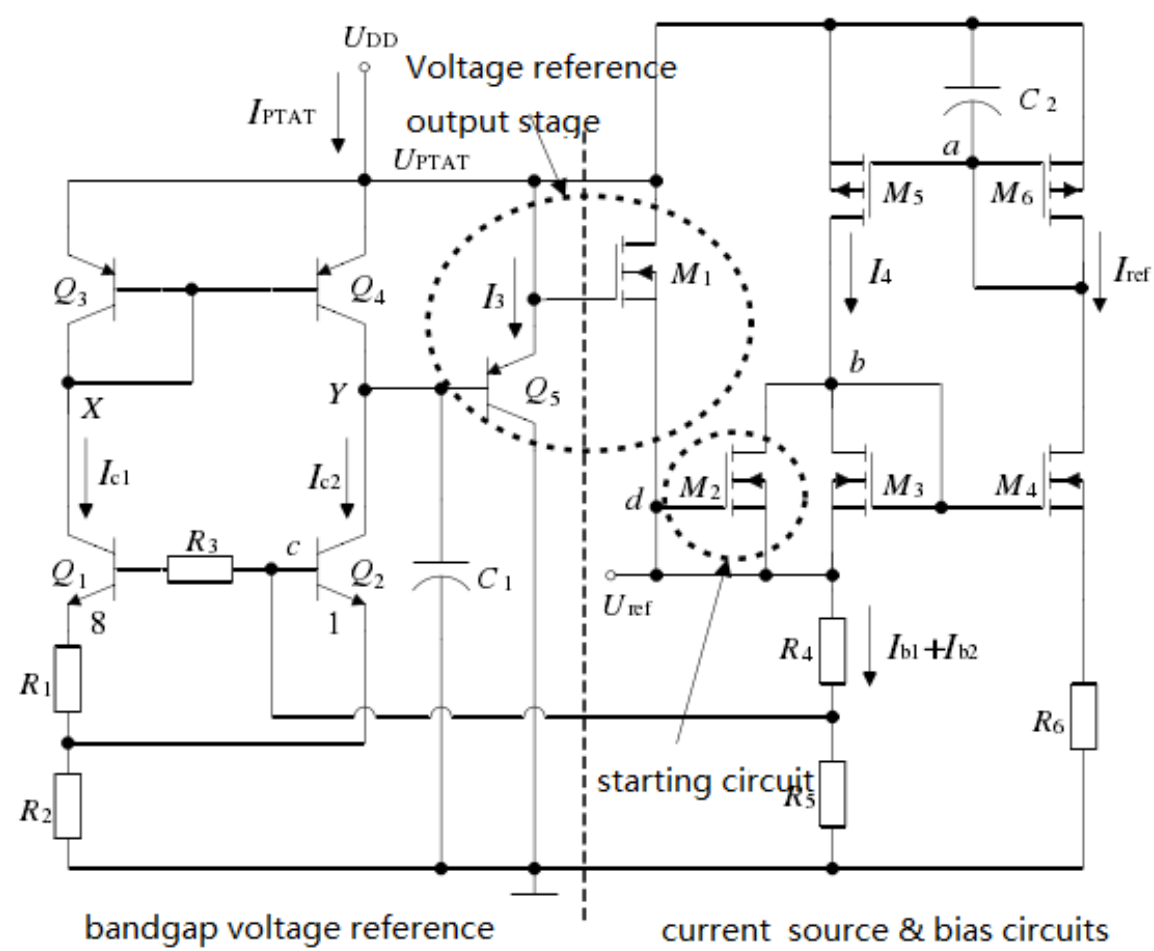

Figure 1: Bandgap reference source and current source core circuit

\section{2) Design of current bias circuit and start circuit}

In the bias circuit of bandgap reference current source, $M_{3}, M_{4}, M_{5}$ and $M_{6}$ commonly constitute the voltage-current conversion circuit. Meanwhile, the capacitance $C_{2}$ connected between the power supply and the gate $M_{5}$ and $M_{6}$ can further improve the performance of the PSR circuit.The width-to-length radio of $M_{4}$ is four times than $M_{3}$, so the reference current $I_{r e f}$ is twice as $I_{4}$.

$$
I_{r e f}=\left(2 \frac{R_{2}}{R_{1}} U_{T} \ln n+U_{B E 2}\right) \frac{2\left(R_{4}+R_{5}\right)}{R_{5} R_{6}}
$$

Here, $R_{6}=625.7 K \Omega$, the reference current is $2 \mu \mathrm{A}$. Since the current reference bias circuit uses a bias structure, the circuit has two steady states: the operating state and the zero-current state. In order to make the system out of the zero-current state at start-up, it is usually chosen to add the starting circuit where the voltage fluctuation is greatest in the two steady states. The design adds a single MOSFET boot module $\left(M_{2}\right)$ in Fig. 3's point b, and whether in terms of structure or performance can meet the normal requirements of the system. It can be seen from the figure that the bipolar transistor $M_{2}$ adopts the equivalent diode connection of connecting the gate and the source. The $M_{2}$ drain is equivalent to the diode cathode, which the gate and the source are equivalent to the anode. The working principle is when the circuit is in zero current state, the a-point voltage pulls up to $U_{D D}$ under the influence of capacitor. At that time, the $M_{6}$ tube is cut-off, and b point voltage is zero, so $M_{2}$ is break over. When the b-point voltage is pulled up to $U_{D D}$, $M_{3}$ and $M_{4}$ are break over., while a-point voltage 
has been pulled low. At this time, $M_{5}$ and $M_{6}$ is break over, and the circuit enter to a normal working state. Due to the rise of b-point voltage, $M_{2}$ is in the cut-off state, and the start circuit close.

\section{3) Design of PTAT current source}

One of the requirements of ideal current reference (low power) is to consume the least amount of bias current. If the PTAT current is used instead of the constant voltage to supply the core power of the bandgap reference source, then the bias current consumption of all the transistors in the reference current output circuit can be zero, and the PSR performance of circuit can be greatly improved by the cascode structure.

In the PTAT current source circuit, $Q_{6}, Q_{7}, Q_{8}$ and $Q_{9}$ commonly constitute the bandgap reference core circuit; current mirror $M_{7}$ and $M_{8}$ ensure that the core circuit has the same collector current, that is $I_{1}=I_{2}$. In order to reduce the power consumption of the PTAT current source circuit, it is necessary to improve the input impedance of the core circuit. The MOSFET impedance working in the saturation region is:

$$
R_{\max }=\frac{U_{S D}}{I_{\max }}=\frac{2 L U_{S D}}{W \mu_{n} C_{o x}\left(U_{G S}-U_{T}\right)}
$$

In the formula, $\mathrm{W}$ is the channel width, $\mathrm{L}$ is the channel length, and $\mu$ is the carrier migration velocity. $C_{o x}$ is the gate parasitic capacitance, $U_{G S}$ is the gate-source voltage difference and $U_{S D}$ is the source-drain voltage difference.

From the formula (12), we can see that the channel length of the MOSFET is proportional to the impedance, so the circuit will design a long channel length for $M_{9}$, which is composed of PMPM with 45 $\mathrm{mp}$ models. In the improvement of circuit PSR performance, the cascode current mirror composed by $M_{9}$, the output impedance of PTAT current is:

$$
R_{o}=g_{m 10} r_{o 10} r_{o 17}
$$

In the formula, $r_{o 10}$ and $r_{o 17}$ are the inner resistance of $M_{10}$ and $M_{17}$, respectively, and $g_{m 10}$ is $M_{10}$ 's transconductance.

The start of PTAT current source is achieved through $M_{18}$. The working principle of whole system works is: when the circuit is in a zero-current state, 0 - point potential is zero.
At that time, if the signal EN can be pulled low, then $M_{18}$ is cut-off and $M_{19}$ is break over.

The voltage will be pulled up to $U_{D D}$ under the state of 0-points divorced from zero current, then the core circuit enter to the normal working state. If the system is limited to produce PTAT current, we just need to pull up the signal EN, then conduction, then the $M_{18}$ is break over and PTAT current source will stop working.

\subsection{Row drive circuit design}

\section{1) Line drive circuit design}

The design of row and column driver for OLED display is shown in Fig. 2. The circuit is an example of the system, and the driver type of other lines is the same as this line. When the line is not scanned, the low level is applied to the line control signal, and then $M_{51}$ is cut-off. The electrical level of this row output high level, and the row is not strobed.

The purpose of connecting the pull-up resistor $R_{20}$ in the circuit is to ensure that each pixel in the row can be reliably turned off in a reverse manner to effectively prevent the cross-effects that may occur on the display screen. If the row is strobed, then the line control signal is connected to the high level so that the $M_{51}$ is break over. The output level of this row is low, at which point each pixel on the row gives out light under the impact of drive current.

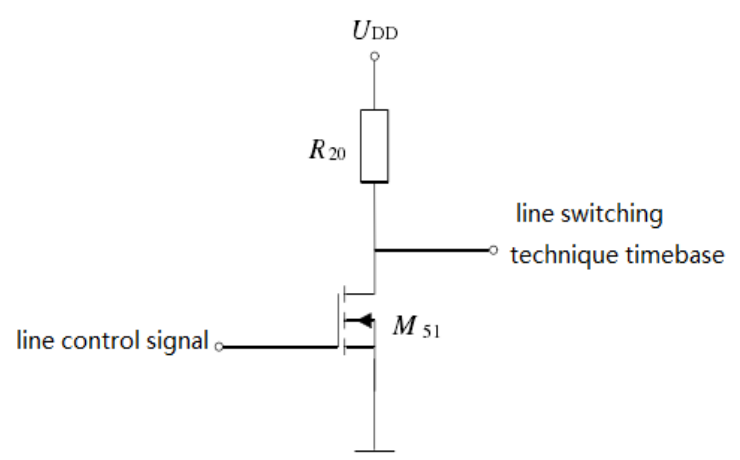

Figure 2: The diagram of line driven circuit

\section{2) Design of column drive circuit}

The drive current of pixel needs a level above the mA. Considering that the fine adjustment of pixel gray scale needs to be done with a small current $(\mu \mathrm{A})$, so the reference current should be copied and amplified. The reference current duplicate circuitry with pixel gray control level of 16 is added for the design of current source. The circuit structure is shown in Fig. 3. 


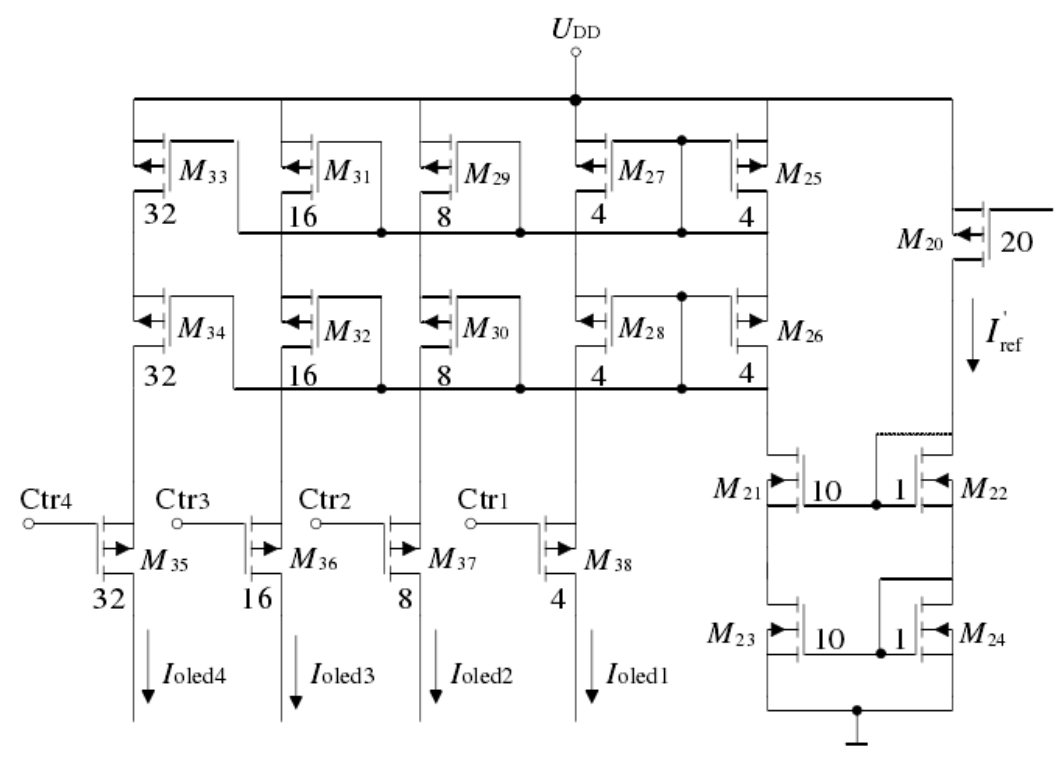

Figure 3: A duplicate circuitry of reference current

In Fig. $3, M_{35}, M_{36}, M_{37}$ and $M_{38}$ is a MOSFET switch tube controlled by digital signals, which mainly controls the pixel influx for the driven current in each row and adjusts the pixel gray.

$C t r_{1}, C t r_{2}, C t r_{3}$ and $\mathrm{Ctr}_{4}$ are the control siginals of four switch tube, respectively. $M_{25}, M_{26}$ ， $M_{27}, \quad M_{28}$ and $M_{21}, M_{22}, M_{23}, M_{24}$ consititutetwo groups mirror current source, respectively. The function of $M_{29}$ and $M_{30}, M_{31}$ and $M_{32}, M_{33}$ and $M_{34}$ is to gradually enlargethe current according to two times of the radio of widthto-length. The base of $M_{20}$ is connected to the drain of $M_{6}$ in core circuit.
Meanwhile, the radio of width-to-length of is 5 times than $M_{6}$. Then the pixel driven current is (we assume that all the characteristics of MOSFETs are same).

$$
I_{\text {oled } 4}=2 I_{\text {oled } 3}=4 I_{\text {oled } 2}=8 I_{\text {oled } 1}=16 I_{\text {ref }}
$$

The drain current of is:

In the formula, $U_{T}$ is threshold voltage, $K=\mu^{C} C_{o x} / 2$. The $U_{T}$ and $\mathrm{K}$ in all of the MOSFETs are equal. Therefore, the driven current value used for adjusting pixel gray is $100 \mu A, 20 \mu A, 400 \mu A$ and $800 \mu A$.

$$
I_{r e f}^{\prime}=80\left(\frac{W_{27} / L_{27}}{W_{25} / L_{15}}\right) \cdot\left(\frac{W_{23} / L_{23}}{W_{24} / L_{24}}\right) \cdot\left(\frac{W_{G S 27}-\mathrm{U}_{T 27}}{W_{G S 25}-\mathrm{U}_{T 25}}\right)^{2} \cdot\left(\frac{W_{G S 23}-\mathrm{U}_{T 23}}{W_{G S 24}-\mathrm{U}_{T 24}}\right)^{2} \cdot\left[\frac{\left(1+\lambda \mathrm{U}_{T 27}\right) K_{27}^{\prime}}{\left(1+\lambda \mathrm{U}_{T 25}\right) K_{25}^{\prime}}\right]\left[\frac{\left(1+\lambda \mathrm{U}_{D S 23}\right) K_{23}^{\prime}}{\left(1+\lambda \mathrm{U}_{D S 24}\right) K_{24}^{\prime}}\right]
$$

\section{The Analysis of Simulation Testing and Results}

In order to measure the performance of changing with the temperature of each drive current, Fig. 4, 5, 6 and 7, respectively shows the characteristic curve of temperature of modulating current $I_{\mathrm{oled} 1}, I_{\mathrm{oled} 2}$, $I_{\text {oled } 3}$ and $I_{\text {oled } 4}$ used to adjust the scale of PMOLED pixel gray.
According to equation (14) calculation, when the temperature changes from $-40^{\circ} \mathrm{C}$ to $125^{\circ} \mathrm{C}$, the temperature coefficient of $I_{\text {oled } 1}, I_{\text {oled } 2}, I_{\text {oled } 3}$ and $I_{\text {oled } 4}$ are $50.2 \mathrm{ppm} /{ }^{\circ}, 50.2 \mathrm{ppm} /{ }{ }_{C}, 50.7 \mathrm{ppm} /{ } \mathrm{C}$ and $50.7 \mathrm{ppm} /{ }^{\circ} \mathrm{C}$. 


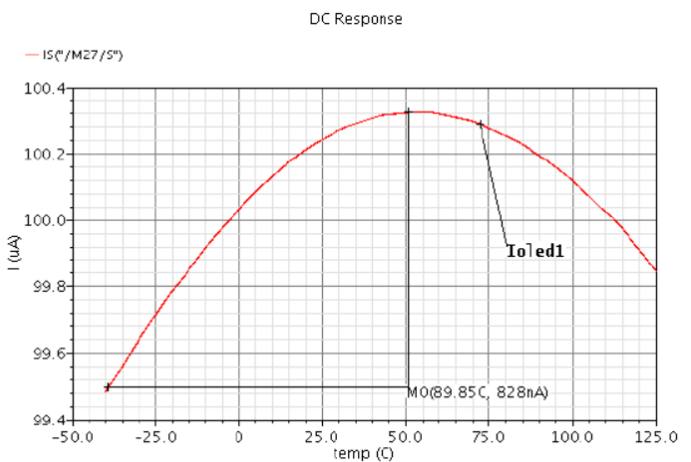

Figure 4: The characteristic curve of temperature 100 of $\mu A$ gray modulation current

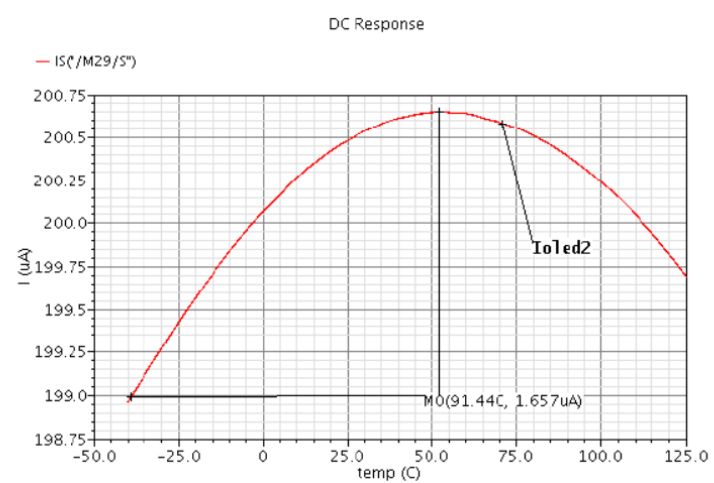

Figure 5: The characteristic curve of temperature 200 of $\mu$ A gray modulation current

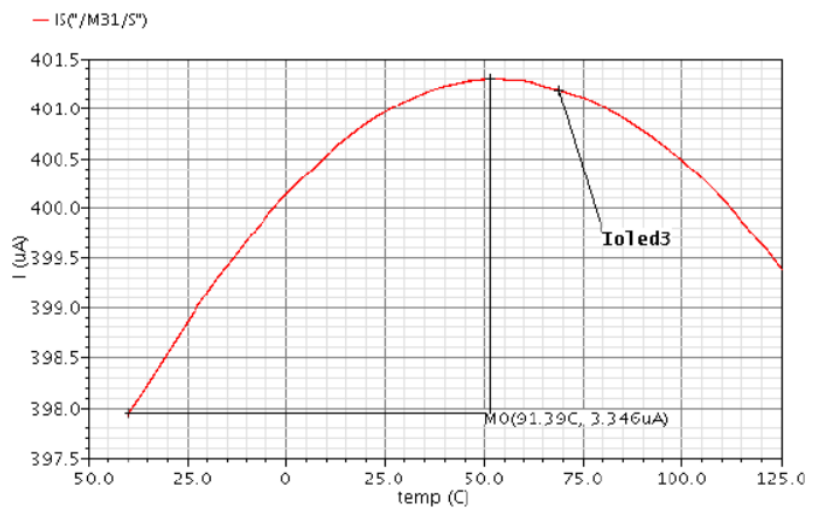

Figure 6: The characteristic curve of temperature 400 of $\mu$ A gray modulation current

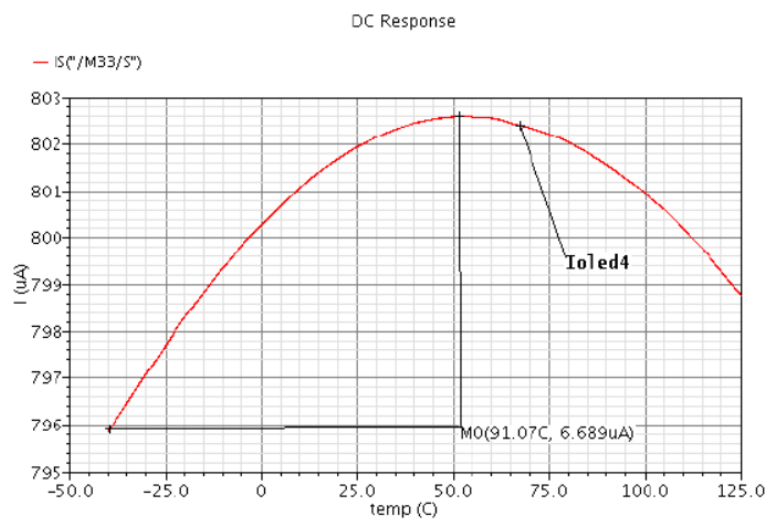

Figure 7: The characteristic curve of temperature 800 of $\mu$ A gray modulation current

According to the above-mentioned drive circuit design flow, the transient response simulation is carried out for the constant current source drive system in the Cadence Analog Design Environment.

Through the simulation test, we can see that all the control signals are high level from 0 to 220 us time, so M35 M38 are in the cut-off state, and the pixel drive current is zero. When the control signal Ctr4, Ctr3, Ctr2 and Ctr1 chaging from high to low in 223 us, 223 us, 423 us and 623 us, the switch tube M35, M36, M37 and M38 is break over in turn. At this time, the driving current of the pixels have increased to $1.2 \mathrm{~mA}, 1.2 \mathrm{~mA}, 1.4 \mathrm{~mA}$ and $1.5 \mathrm{~mA}$ in turn.
The simulation results show that the PMOLED display can work normally on the drive system centered on the constant current source, and the driving current with high precision completely guarantees the brightness uniformity of the display screen, thus improving the service life of the display screen.

\section{Conclusion}

The pixel drive current with high-precision, high stability and low temperature coefficient has a directly impact on y brightness uniformity, pixel 
aging, cross-effects of PMOLED display. Based on this, this paper designs the PMOLED display drive system based on PAM, and simulates the PMOLED display drive system based on constant current source. The test results show that the PMOLED display can be used in the drive system concered on constant current source. The driving current generated by the bandgap reference source has the characteristics of high precision, low temperature coefficient and high stability. Under the action of the line circuit control signal, the PMOLED display pixel drive current can accurately increase from $1.2 \mathrm{~mA}$ to $1.4 \mathrm{~mA}$ and $1.5 \mathrm{~mA}$, which ensures the uniformity of the display brightness, thereby improving the display life.

\section{Reference}

[1]Lu Guizhu (College of Electron Engineering, Dongguan University of Technology, Dongguan 523808,CHN); Research on OLED Color Display Driver Technology. [J]. Semiconductor Optoelectronics, 2011-02.

[2]J. Jacobs, D. Hente, and E. Waffenschmidt, "Drivers for OLEDs," in IEEE Industry Applications Conference, pp. 1147-1152, 2007.
[3] J. Betts-LaCroix, Selective Dimming of OLED displays. US Patent 0149223 A1, 2010.

[4] W.-B. Lee, K. Patel, and M. Pedram, "White-LED backlight control for motion-blur reduction and power minimization in large LCD TVs,"[J]. of SID, vol. 17, no. 1, pp. 37-45, 2009.

[5]M. Kimufa, Y. Hara, H. Hara, T. Okuyama, S. Inoue, T. Shimoda, "Classification of driving methods for TFT-OLEDs and novel proposal using time ratio grayscale and current uniformization", IEICE Trans. Electron., vol. E88-C, no. 11, Nov. 2005.

[6]Y. O. Nam and et al., "Power-Constrained Contrast Enhancement Algorithm Using Multiscale Retinex for OLED Display," Image Processing, IEEE Trans. on, vol. 23, no. 8, pp. 3308 3320, Aug. 2014.

[7]C. H. Lin and et al., "Catch your attention: Quality-retaining Power Saving on Mobile OLED Displays," in Proc. of DAC 14, Jun. 2014, pp. 1-6.

[8]K. W. Tan and et al., "FOCUS: A Usable \& Effective Approach to OLED Display Power Management," in Proc. of UbiComp 13, Sep. 2013, pp. 573-582. 